\title{
A STUDY ON THE POST SURGICAL WOUND INFECTIONS IN A TERTIARY CARE HOSPITAL IN KANCHIPURAM
}

\author{
Sivasankari Selvaraj1, Thenmozhi Valli Pitchai Rathinam², Anitha Chandrahausan³, Senthamarai Srinivasan4, Ve \\ ${ }_{1}^{1}$ Associate Professor, Department of Microbiology, Meenakshi Medical College Hospital and Research Institute. \\ ${ }^{2}$ Dean, Meenakshi Medical College Hospital and Research Institute. \\ ${ }^{3}$ Assistant Professor, Department of Microbiology, Meenakshi Medical College Hospital and Research Institute. \\ 4 Professor, Department of Microbiology, Meenakshi Medical College Hospital and Research Institute. \\ ${ }^{5}$ Director of PG Studies, Meenakshi Medical College Hospital and Research Institute.
}

ABSTRACT

\section{BACKGROUND}

Surgical site infections are the infections that occur within thirty days after the operative procedure (Except in case of added implants). Surgical site infections are the $3^{\text {rd }}$ most commonly reported nosocomial infections accounting for a quarter of all such infections. A wide range of organisms are known to infect wounds like gram positive cocci, gram negative bacilli, spore formers, aerobes and anaerobes. Despite the advances in operative technique and better understanding of the pathogenesis of wound infections and wound healing, surgical site infections still remain a major source of morbidity and mortality. Hence, this study was done to identify the aetiological bacterial agents and their antibiogram pattern and the risk factors associated with surgical site infections.

\section{METHODS}

Wounds were examined for signs and symptoms of infection in postoperative ward. All the pus swabs were processed and identified as per standard methods of identification. Antibiogram was performed as per CLSI guidelines. The isolates were screened and confirmed with double disc diffusion method using CLSI guidelines.

\section{RESULTS}

The rate of surgical site infections in our study was $8.3 \%$. The rate of surgical site infections was higher (73.3\%) in emergency surgeries than the elective surgeries. E. coli was the commonest isolate among gram negative bacilli; $33.3 \%$ isolates of E. coli were ESBL procedures. E. coli were sensitive to cefepime and ciprofloxacin and showed maximum resistance to ampicillin and ceftazidime. All the E. coli were sensitive to imipenem.

\section{KEYWORDS}

Surgical Site Infections, Nosocomial, Surgeries, E. coli, ESBL.

HOW TO CITE THIS ARTICLE: Selvaraj S, Rathinam TVP, Chandrahausan A, et al. A study on the post surgical wound infections in a tertiary care hospital in Kanchipuram. J. Evolution Med. Dent. Sci. 2016;5(22):1180-1183, DOI: 10.14260/jemds/2016/274

\section{INTRODUCTION}

Surgical site infections are those infections, which occurs in the surgical incisions and involving structure adjacent to the wounds exposed during surgery. ${ }^{1}$ Surgical site infections are one of the major health problems that occur throughout the world accounting for $20-25 \%$ of nosocomial infections worldwide. ${ }^{2}$ These infections are caused by exogenous and endogenous microorganisms that enter the operative wound during the procedure. ${ }^{3}$ Surgical site infections occurs within 30 days after the postoperative procedure (Except in cases of added implants, where the duration can extend upto 1 year from operation). Surgical site infections can occur any time from zero to thirty days affect either the incision or the deep tissue at the operation site. ${ }^{4}$ Globally, surgical site infection rates have been reported as $2.5 \%$ to $41.9 \% .{ }^{5}$ In India, the rate of surgical site infections ranges from $4-30 \% .^{6}$ The incidence of infected surgical wound is associated the incidence of

Financial or Other, Competing Interest: None.

Submission 03-02-2016, Peer Review 27-02-2016,

Acceptance 05-03-2016, Published 16-03-2016.

Corresponding Author:

Dr. Sivasankari Selvaraj,

Associate Professor,

Dept. of Microbiology,

Meenakshi Medical College Hospital \& Research Institute,

Enathur, Kanchipuram.

E-mail: murugansivasankari1@gmail.com

DOI: $10.14260 /$ jemds $/ 2016 / 274$ infected surgical wound is associated with risk factors such as preoperative care, theatre environment and postoperative care. The common cause for wound disruption are overweight, increasing age, poor nutrition, jaundice, diabetes, smoking, malignancy, presence of prior scar or radiation at the wound site. $^{7}$

The common pathogenic bacteria in surgical site infections include Staphylococci, Pseudomonas, Streptococci, Enterococci, E. coli, Klebsiella, Enterobacter, Citrobacter, Acinetobacter, Proteus and S. aureus. S. aureus is present as a normal flora, can be isolated upto $60 \%$ from nose and can be readily transmitted from person to person. ${ }^{3}$ Surgical site infection is the index of the health care system of any hospital. With the increase in incidence of nosocomial infections and multidrug resistance, a meticulous and periodic surveillance of various hospital acquired infections is called for.

With an active infection control team operating in the hospital, surgical site infections are naturally one of the topmost priorities on the agenda; hence, the following study was undertaken. Diagnosis was made according to the criteria established by CDC Atlanta. ${ }^{8}$ Hence, this study was done to identify the etiological bacterial agents, antibiogram pattern and the risk factors causing surgical site infections.

\section{MATERIALS AND METHODS}

This study was conducted in a tertiary health care centre in Kanchipuram during the period of Jan to Nov 2015. The study 
population included 30 patients suffering from surgical site infections out of a total of 360, who have undergone clean contaminated surgeries and clean surgeries during the tenure of the study.

\section{Inclusion Criteria}

A clean contaminated and clean surgery infected with a pus discharge. Signs of sepsis (Warmth, Tenderness, Erythema, Indurations).

\section{Exclusion Criteria}

Wounds other than surgical wound, stitch abscess, no drainage, burns contaminated and dirty wounds.

All patients were well informed about the study and informed consent was obtained. Wounds were examined for signs and symptoms of infection in postoperative ward. When infection was suspected, the area around the wound was cleaned with saline. Exudates were collected from depth of the wound using 2 sterile swabs. A short clinical history regarding the age, sex, type of illness, diagnosis, type of surgery performed. Antibiotics given were noted.

Wound was evaluated on $3^{\text {rd }}$ day, $5^{\text {th }}$ day and till discharge. Patients were followed up for 30 days after surgery to identify any infection at surgical site. All the pus swabs were processed and identified as per standard methods of identification. Antibiogram was performed as per Clinical and Laboratory Standards Institute (CLSI) guidelines. Antibiotics used included ampicillin, gentamicin, ciprofloxacin, ofloxacin, imipenem, ceftriaxone, cefepime, ceftazidime, cefazolin and cefotaxime.

The antibiotic discs were obtained from Hi-Media Laboratories were used. Isolates of $E$. coli were screened for Extended Spectrum $\beta$ Lactamase (ESBL) production using ceftazidime (30 $\mathrm{mg}$ ) and cefotaxime (30 $\mathrm{mg}$ ) and ceftriaxone (30 mg). The isolates were confirmed with double disc diffusion method using Clinical and Laboratory Standards Institute (CLSI) guidelines. ${ }^{9}$

\section{RESULTS}

A total of 360 patients were operated during the study period. Out of which 30 patients who developed surgical site infections were enrolled in the study. Out of the 30 patients, 17 (56.6\%) were males and 13 (43.3\%) were females. Infection rate was $8.3 \%$.

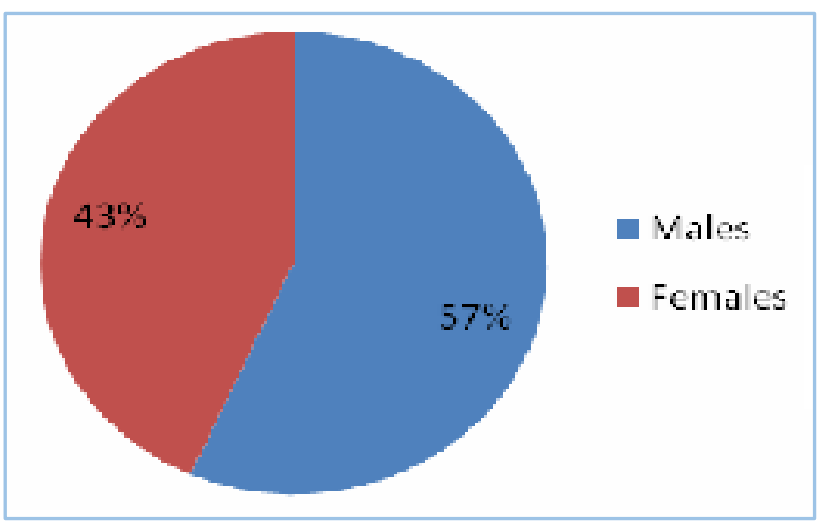

Among the cases, studied emergency procedures were $22(73.3 \%)$ and elective procedures were 8 (26.6\%).

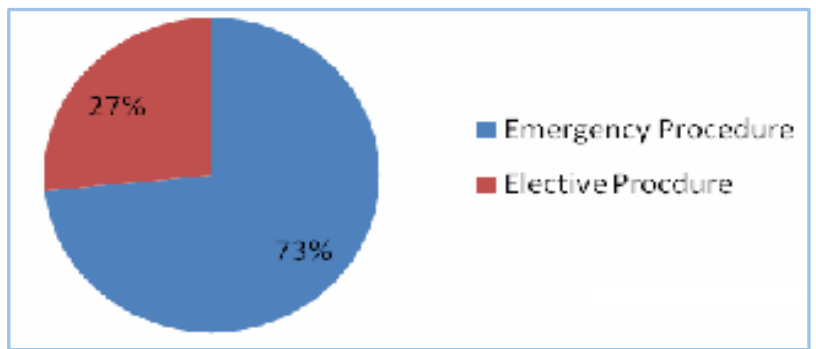

In the cases included, $14(46.6 \%)$ were clean surgeries and $16(53.3 \%)$ were clean contaminated surgeries.

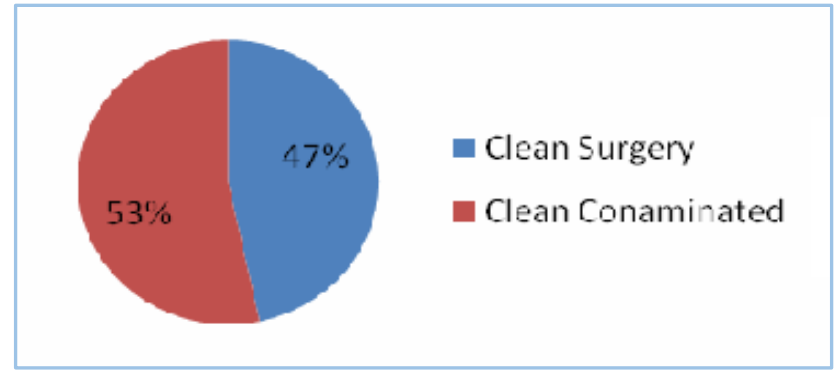

Out of 30 patients, 23 (76.6\%) patients showed significant growth and 7 (23.3\%) patients showed no growth.

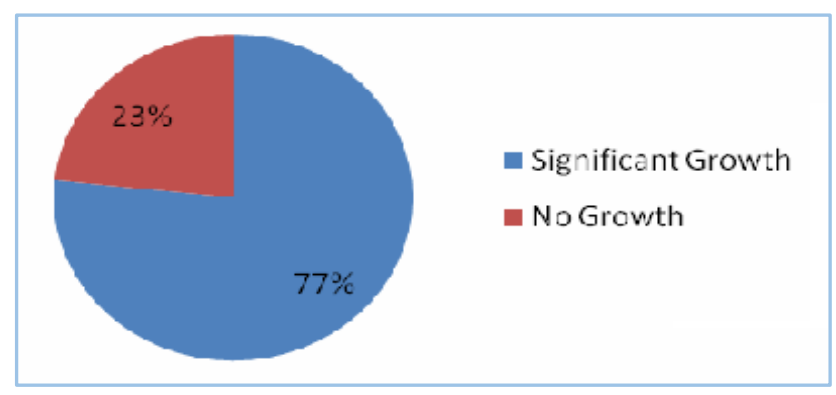

\begin{tabular}{|c|c|c|c|}
\hline $\begin{array}{c}\text { Duration of } \\
\text { Surgery }\end{array}$ & No. & $\mathbf{\%}$ & $\begin{array}{c}\text { Culture } \\
\text { Positivity }\end{array}$ \\
\hline $0-1$ hrs. & 8 & 26.6 & 3 \\
\hline $1-2$ hrs. & 10 & 33.3 & 8 \\
\hline$>2$ hrs. & 12 & 40.1 & 12 \\
\hline \multicolumn{3}{|c|}{$\begin{array}{r}\text { Table 1: Correlation between Duration } \\
\text { of Surgery with Culture Positivity }\end{array}$} \\
\hline
\end{tabular}

\begin{tabular}{|c|c|c|}
\hline Risk Factor & No. & \% \\
\hline Diabetes & 18 & 60.1 \\
\hline Hypertension & 4 & 13.3 \\
\hline Diabetes + Hypertension & 2 & 6.6 \\
\hline Smoking & 5 & 16.6 \\
\hline Alcohol & 7 & 23.3 \\
\hline \multicolumn{2}{|c|}{ Table 2: Showing Patients with } \\
Underlying Risk Factors \\
\hline
\end{tabular}

Diabetes is the commonest risk factor followed by alcohol consumption. 


\begin{tabular}{|c|c|c|}
\hline Organisms & No. & \% \\
\hline Staphylococcus aureus & 5 & 17.5 \\
\hline Enterococcus sp & 2 & 7.4 \\
\hline E. coli & 6 & 21.2 \\
\hline Klebsiella & 4 & 14.8 \\
\hline Pseudomonas sp & 5 & 17.5 \\
\hline Proteus mirabilis & 2 & 7.4 \\
\hline Acinetobacter sp & 3 & 11.1 \\
\hline \multicolumn{2}{|c|}{ Table 3: Organisms Isolated in } \\
\hline
\end{tabular}

Out of 23 samples which showed growth, 27 organisms were isolated.

E. coli was the predominant organism followed by $S$. aureus.

Among 23 culture positive samples, 19 (70.37\%) yielded single organism and 4 (14.8\%) yielded dual organism.

\begin{tabular}{|c|c|c|}
\hline Antibiotics & No. & $\%$ \\
\hline Ampicillin & 6 & $100 \%$ \\
\hline Gentamycin & 4 & 66.6 \\
\hline Ciprofloxacin & 2 & 33.3 \\
\hline Ceftriaxone & 3 & 50.1 \\
\hline Cephazolin & 3 & 50.1 \\
\hline Ceftazidime & 5 & 83.3 \\
\hline Cefotaxime & 4 & 66.6 \\
\hline Cefepime & 1 & 17.6 \\
\hline $\begin{array}{l}\text { Piperacillin } \\
\text { tazobactam }\end{array}$ & 1 & 17.6 \\
\hline Imipenem & Nil & - \\
\hline \multicolumn{3}{|c|}{ Table 4: Resistance Pattern of E. coli } \\
\hline
\end{tabular}

Maximum resistance was seen in Ampicillin and Ceftazidime, while the least resistance was with reference to Cefepime. All isolates were sensitive to Imipenem. Among the 6 isolates of E. coli, ESBL, detection was done and 2 (33.3\%) isolates were ESBL producers.

\section{DISCUSSION}

The rate of surgical site infections greatly varies worldwide and from hospital to hospital from $2.5 \%$ to $41.9 \% .{ }^{10}$ In our study the infection rate was $8.3 \%$, which is slightly higher than Anvikar et al. (6.09\%), but much lower than Patel et al. who reported $16 \%$ of surgical site infections. ${ }^{11,12}$

In our study, 17 (56.6\%) mere males and 13 (43.3\%) were females. Similarly, Mawalla et al. reported male preponderance of surgical site infections 39 (60\%) over females $26(40 \%) .^{2}$ This could be due to cigarette smoking in males. Smoking has an impact on wound healing through impairment of tissue oxygenation local hypoxia via vasoconstriction. ${ }^{13}$

In our study, the rate of surgical site infections was higher in emergency surgeries, $73.3 \%$ than in elective surgeries $26.6 \%$. This study was concordant with Patel et al. who also reported a similar preponderance of infections in emergency surgeries (24.1\%) over elective surgeries $(12.6 \%) .12$

Emergency surgeries are often done by juniors and duty doctors and there may be a little time for preoperative procedures. $^{11}$ In our study, the surgical site infections incidence in clean surgeries was $14(46.6 \%)$ and clean contaminated surgeries was $16(53.3 \%)$. This is much higher than Madhusudhan et al. who reported $12 \%$ in clean contaminated and $11 \%$ in clean surgeries. ${ }^{14}$ The rate of surgical site infections varies from surgeon to surgeon. The skill and experience also affect the degree of contamination of surgical site through breaks in technique. ${ }^{12}$

In our study, 23 (76.6\%) patients showed significant growth which was slightly lower than Neelesh Naik et al. who also reported $81.1 \%$ of significant growth in their study. ${ }^{15}$ In our study, $23.3 \%$ showed no growth which is slightly higher than Neelesh Naik et al. who showed $18.2 \%$ samples to be culture negative. Culture negativity may be due to antibiotic therapy prior to culture of material from an apparently infected site. 16

In our study, the surgeries performed for more than 2 hrs. yielded culture positive and the rate of surgical site infections was also as high as $40.1 \%$. This is concordant with Varsha et al. who also reported higher culture positivity in longer duration of surgeries $12.5 \% .17$ Prolonged duration surgeries results in increased exposure of operation site to air, prolonged trauma, stress of prolonged anaesthesia and sometimes blood loss. ${ }^{18}$

In our study, diabetes constitutes an important predisposing factor for surgical site infections followed by alcoholism and smoking. This is concordant with study done by Ramesh et al. who also reported diabetes and alcohol as important risk factors for surgical site infections. ${ }^{19}$ Similar findings were reported by S.M. Patel et al. who reported 36.4\% of surgical site infections in patients with diabetes mellitus. ${ }^{12}$

In our study among 23 culture positive isolates, 19 $(70.37 \%)$ yielded single organism. This is lower than Neelesh Naik et al. who reported a corresponding yield of $92.16 \%$. In our study, $4(14.8 \%)$ dual organisms which is much higher than Neelesh Naik et al. who reported $7.84 \%$ of dual organisms causing surgical site infections. ${ }^{15}$

S. aureus is the predominant isolate among gram positive and in gram negative E. coli was the most commonly isolated. This is concordant with Ramesh et al. who has also isolated a similar pattern of organisms. ${ }^{19}$

It has been found that in clean surgeries, S. aureus from the exogenous environment or the patient's skin flora is the usual pathogen. Whereas in other categories of surgical procedure clean contaminated the polymicrobial flora closely resembling the normal endogenous microflora of the affected site is the frequently isolated pathogen. ${ }^{20}$

The organisms most frequently involved in surgical site infections change from time to time and from place to place and so does their sensitivity to various antibiotics. ${ }^{21}$

Among the gram negatives, E. coli was the commonest isolate and was sensitive to cefepime, ciprofloxacin and showed maximum resistance to ampicillin and ceftazidime. All the E. coli isolates were sensitive to imipenem.22,23,24

Among the 6 isolates of E. coli, ESBL detection was done and $2(33.3 \%)$ isolates were ESBL producers. This is much higher than Saraswathi et al. who had isolated $14.2 \%$ of ESBL in their study. ${ }^{25}$ Findings from this study on surgical site infections suggests that the introduction of evidence based antibiotic policy in the hospital is a must.

\section{CONCLUSION}

The rate of surgical site infections in our study was $8.3 \%$. The rate of surgical site infections were high in emergency surgeries $73.3 \%$ as compared to elective surgeries. The incidence of surgical site infections was more (40.1\%) in long duration surgeries performed for more than 2 hrs. Diabetes 
constitutes an important predisposing factor followed by smoking and alcohol.

E. coli was the commonest isolate among gram negative bacilli and 33.3\% isolates of E. coli were ESBL procedures.

Surgical site infections were more in diabetic populations. This indicates that underlying risk factor needs to be carefully evaluated in treating surgical site infections.

Infection control measures such as active surveillance of surgical site infections, compliance observation and instruction/training of healthcare workers, adherence to preoperative antibiotic prophylaxis, maintenance of normothermia and blood glucose control are essential in order to prevent surgical site infections.

\section{REFERENCES}

1. Patherick ES, Dalton JE. Methods for identifying surgical wound infections after discharging from hospital, a systematic review. BMC Infects 2006;6:170-78.

2. Brain Mawalla, Stephen E Mshana, Philipo L Chaly. Predictors of surgical site infections among patients undergoing major surgery at Buganda medical centre in northwest Tanzania. BMC Surgery 2011;11:21.

3. Nichols RL. Current strategies for prevention of surgical site infections. Curr infect Dis Rep 2004;6(6):426-434.

4. Dana KA, Timothy RB, David LD, et al. Schally principles of surgery. New York, USA, McGraw Hill companies. Chapter 6,2010 ; $9^{\text {th }}$ ed:113-133.

5. Olsonmm, Lee JT. Continuous 10 years wound infection surveillance, result advance and unanswered questions. Arch Surg 1990;125:794-803.

6. Mustafa A, Bukhari A, Kakru DK, et al. Incidence of nosocomial mound infection in post-operative patients. JK Practitioner 2004;11:38-40.

7. Vidyadhar B Bangal, Sai K Borawake, Kunanda Shinde. Study on surgical site infections at tertiary care teaching hospital in rural India. International Journal of Biomedical Research 2014;5(2).

8. Deverick J Anderson. Surgical site infections. Infect Dislin N Am 2011;25:135-153.

9. Varaiya A, Kulkarni M, Bhalckar P, et al. Incidence of metllo beta lactamase producing pseudomonas aceuroginosa in diabetes and cancer patients. Indian J Pathol Microhiol 2008;51(2):200-3.

10. Lilami SP, Jangale N, Chowdhary A, et al. Surgical site infection in clean \& clean contaminated cases. Indian J Medical Microbiology 2005;23(4):249-252.

11. Anvikar AR, Deshmukh Ans, Kayakarte RP, et al. A one year prospective study of 3280 surgical wounds. Indian J Medical Microbiological 1999;17(3):129-132.
12. Patel SM, Kinariwala DM, Paul SD, et al. Study of risk factors including NNIS risk index in surgical site infections in abdominal surgeries. Gujarat E Medical journal 2011;66(1).

13. Jone SK, Tripliff RG. Relationship of cigarette smoking to impaired infra oral wound healing: a review evidence and implication for patient care. J Oral Maxilla Surgery 1992;50:237-40.

14. Madhusudhan NS, Mareena Thomas. Study of surgical site infections in clean \& clean contaminated surgeries in a tertiary care hospital. International journal of biomedical research 2014;5(8).

15. Neelesh R Naik, Rama NK, Sharadhadevi Mannur. Antibiotic susceptibility pattern of bacterial isolates causing surgical site infections. National Journal of Basic Medical Science 2012;3(2).

16. Danure MA, Cohen EJ, Sudesh S, et al. Spectrum of fungal keratitis at wills eye hospital, philadelphia, pennsylvania. Cornea 2000;19(3):307-312.

17. Varsha Shahene, Upendra Lele. Surgical site infections, a one year prospective study in a tertiary care center. Qa Surgical site infections on university, International journal of health sciences 2012;6(1).

18. Tripathy BS, Roy N. Post-operative would infections. J Indian MA 1975;64(4):90-3.

19. Ramesh A, Dharni R. Surgical site infections in a teaching hospital clinco microbiological and epidemiological profile. Int I Boil Med Res 2012;3(3):2050-2053.

20. Richard PE. Surgical site infections preventation and control: an emerging paradigm. J Bone Joint Surg Am 2009;91:2-9.

21. Murthy R, Sengupta S, Maya N, et al. Incidence of postoperative around infection and their antibiogram in a teaching hospital. Indian J Med Sci 1998;52:553-5.

22. Nandita Pal, Rajyasri Guhathakurta (Mukherjee). Surgical site infection in surgery ward at a tertiary care hospital: the infection rate and the bacteriological profile. IOSR Journal of Pharmacy 2012;2(5):01-05.

23. Bemnet Amare, Zeki Abdurrahman, Beyene Moges, et al. Postoperative surgical site bacterial infections and drug susceptibility patterns at Gondar university teaching hospital, northwest ethiopia. J Bacteriol Parasitol 2011;2:8.

24. Amrita R Bhadauria, Chella Hariharan. Clinical study of postoperative wound infections in obstetrics and gynaecological surgeries in a tertiary care set up. Int J Reprod Contracept Obstet Gynecol 2013;2(4):631-638.

25. Saraswathi R, Velayutharj A, Shailesh Kumar. Prevalence of pathogenic microbes in post-operative wound infection in various surgical specialties. International Journal of Developmental Research 2014;4(8):1783-1786. 